\title{
Diffraction and geometrical optical transfer functions: calculation time comparison
}

José Antonio Díaz, Virendra N. Mahajan

José Antonio Díaz, Virendra N. Mahajan, "Diffraction and geometrical optical transfer functions: calculation time comparison," Proc. SPIE 10375, Current Developments in Lens Design and Optical Engineering XVIII, 103750D (23 August 2017); doi: 10.1117/12.2275377

EDent: SPIE Optical Engineering + Applications, 2017, San Diego, California, United States 


\title{
Diffraction and geometrical optical transfer functions: calculation time comparison \\ José Antonio Díaz ${ }^{\mathrm{a}^{*}}$ and Virendra N. Mahajan ${ }^{\mathrm{b}}$ \\ a Departamento de Óptica, Universidad de Granada, 18071-Granada, Spain \\ ${ }^{\mathrm{b}}$ The College of Optical Sciences, University of Arizona Tucson, AZ 85721
}

\begin{abstract}
In a recent paper, we compared the diffraction and geometrical optical transfer functions (OTFs) of an optical imaging system, and showed that the GOTF approximates the DOTF within $10 \%$ when a primary aberration is about two waves or larger [Appl. Opt., 55, 3241-3250 (2016)]. In this paper, we determine and compare the times to calculate the DOTF by autocorrelation or digital autocorrelation of the pupil function, and by a Fourier transform (FT) of the point-spread function (PSF); and the GOTF by a FT of the geometrical PSF and its approximation, the spot diagram. Our starting point for calculating the DOTF is the wave aberrations of the system in its pupil plane, and the ray aberrations in the image plane for the GOTF. The numerical results for primary aberrations and a typical imaging system show that the direct integrations are slow, but the calculation of the DOTF by a FT of the PSF is generally faster than the GOTF calculation by a FT of the spot diagram.
\end{abstract}

Keywords: Optical transfer function, aberrations, geometrical OTF, diffraction OTF, imaging.

\section{INTRODUCTION}

In our earlier work $[\mathbf{1 , 2}]$, we discussed and compared the diffraction and geometrical OTFs, and gave the threshold value of a primary aberration above which the GOTF is equal to the DOTF within $5 \%$ or $10 \%$ over a spatial frequency range of interest where the DOTF falls to a value of 0.1. Our motivation for such comparison was based on the general notion that it was easier and faster to calculate the GOTF, which, in turn, offered an advantage for repetitive OTF calculations when tolerancing a design of an optical imaging system with large aberrations at various optical wavelengths and field angles.

Major optical design software such as ZEMAX [3], OSLO [4], SYNOPSIS [5] and CODE V [6] allow calculating the DOTF by FT of the PSF, and by pupil function autocorrelation [7]. GOTF is calculated as the FT of the spot diagram [8]. In this paper, we examine and compare the times for calculating the diffraction and geometrical OTFs. We calculated these OTFs for primary aberrations at their threshold values given in Table 3 in Ref. [1,2]. We also consider a typical optical imaging system design from the literature. Based on such numerical examples, we conclude that the calculation time for the DOTF by FT of the PSF is faster than that of the GOTF by FT of the spot diagram. In conjunction with the fact that GOTF is an approximation of DOTF for only large aberrations, whose magnitude varies with their type or the orientation of the spatial frequency, the lack of faster GOTF calculation eliminates the need to consider it in optical design.

\footnotetext{
* Corresponding author: jadiaz@ugr.es
} 


\section{BACKGROUND}

The DOTF, $\tau_{d}(\xi, \eta)$, of an imaging system is given by the autocorrelation of its pupil function, which can be written $[2,7]$

$$
\tau_{d}(\xi, \eta)=\frac{1}{\pi} \iint \exp \{2 \pi i Q(x, y ; \xi, \eta)\} \mathrm{d} x \mathrm{~d} y
$$

where

$$
Q(x, y ; \xi, \eta)=W(x+\xi, y+\eta)-W(x-\xi, y-\eta)
$$

is the aberration difference function for a spatial frequency $(\xi, \eta)$ and the region of integration is the overlap area of the two mutually displaced pupils. Here, $W(x, y)$ is the wave aberration function in units of wavelength $\lambda,(x, y)$ are the coordinates of a pupil point normalized by the pupil radius $a$, and $(\xi, \eta)$ is the spatial frequency in units of the diffraction cutoff frequency $1 / \lambda F$ ( $F$ being the focal ratio of the image-forming light cone). It should be evident that the starting point for the DOTF calculation is the wave aberration function, which is obtained in the optical design process by ray tracing.

The aberrated GOTF, $\tau_{g}(\xi, \eta)$ corresponding to a spatial frequency $(\xi, \eta)$ is given by $[\mathbf{2 , 7 ]}$

$$
\tau_{g}(\xi, \eta)=\frac{1}{\pi} \iint_{x^{2}+y^{2} \leq 1} \exp \left[2 \pi i\left(\xi x_{i}+\eta y_{i}\right)\right] \mathrm{d} x \mathrm{~d} y
$$

where $\left(x_{i}, y_{i}\right)$ are the transverse ray aberrations in units of $\lambda F$ given by [9]

$$
\left(x_{i}, y_{i}\right)=2\left(\frac{\partial W}{\partial x}, \frac{\partial W}{\partial y}\right)
$$

Although the transverse ray aberrations can be obtained from the wave aberration function, they can be obtained simply by tracing the rays up to the image plane. Indeed that is how the spot diagram is obtained. A simpler approach to obtain the GOTF is to FT the spot diagram [8]:

$$
\tau_{g}(\xi, \eta)=\frac{1}{N} \sum_{i=1}^{N} e^{-2 \pi i\left(\xi x_{i}+\eta y_{i}\right)}
$$

where $N$ is the number of spots obtained by tracing rays. Although a spot diagram does not represent the geometrical PSF exactly, our numerical results indicate that the GOTF obtained in this manner is very close to that obtained from Eq. (3). 


\section{METHODS OF OTF CALCULATION}

All of the calculations were performed in a $3.4 \mathrm{GHz}$ PC desktop with an Intel i7 QuadCore, having $16 \mathrm{~Gb}$ of RAM, and Windows 7 OS (64 bits). Commercial software Mathematica ${ }^{\mathrm{TM}}$ [10] was used for the numerical computations.

\subsection{Numerical calculation of DOTF}

We calculated the DOTF by applying the following numerical algorithms:

1. Numerical integration (NI) of Eq. (1): The values of the integral were obtained in the tangential, sagittal, and $45^{\circ}$ directions by sampling the spatial frequency in the interval $\left.0 \leq \sqrt{\xi^{2}+\eta^{2}}=\nu \leq 1\right)$ comprising 64 points.

2. Digital autocorrelation (DA) of the pupil function: The pupil function was sampled at $64 \times 64$ points yielding $64 \times 64$ DOTF values in the spatial frequency domain $0 \leq \nu \leq 1$.

3. FT of the PSF: Again, the pupil function was sampled at $64 \times 64$ points yielding $64 \times 64$ DOTF values in the spatial frequency domain for which $0 \leq \nu \leq 1$.

Any aliasing was avoided by setting the appropriate pupil sampling so that the DOTF numerical results by DA and FT of the PSF agree with those obtained by NI, and checking that by increasing the pupil function sampling did not change those results.

\subsection{Numerical calculation of GOTF}

The GOTF was calculated by applying the following numerical algorithms:

1. NI of Eq. (3): The values of the integral were obtained in the tangential, sagittal, and $45^{\circ}$ directions by sampling the spatial frequency in the interval $0 \leq \nu \leq 1$ comprising 64 points.

2. FT of the spot diagram: The pupil was sampled at $32 \times 32$ points, resulting in a spot diagram of 797 spots.

\section{NUMERICAL EXAMPLES}

\subsection{Primary aberrations}

We calculated the DOTF and GOTF for defocus, astigmatism, coma, spherical aberration balanced for minimum wave aberration standard deviation $\sigma_{w}$, and that balanced for minimum ray aberration standard deviation or spot sigma $\sigma_{s}$. The aberration values were the same as those in Ref. [1,2], as also shown in Table 1.

\subsection{Imaging System}

The OTFs were also calculated for an $\mathrm{f} / 2.5$ double Gauss lens designed for use in a motion-picture camera (35-mm film) [11], working at a wavelength of $546.1 \mathrm{~nm}$, on-axis and at a field angle of $12^{\circ}$, in the tangential and sagittal directions. We checked that the exit pupil was basically circular, and thus wavefront fitting by using Zernike polynomials allowed the software to interpolate at non-uniform sampling grids when applying quadrature rules in the numerical integrations. 


\section{NUMERICAL RESULTS}

\subsection{Primary aberrations}

We investigated several integration rules, and found that, the "GaussKronrod" rule [12] was the fastest for DOTF calculations, and "MultiPeriodic" for the GOTF calculation. Table 1 shows the calculation times for obtaining the two OTFs by NI of Eqs. (1) and (3), respectively, DA of the pupil function, FT of the PSF, and FT of the spot diagram. Contrary to the general notion, the calculation of GOTF is not faster than that of the DOTF. Overall, the fastest way to calculate the DOTF is by FT of the PSF, and by FT of the spot diagram for the GOTF. We note that even NI of Eq. (1) for the DOTF is faster than the NI of Eq. (3) for the GOTF.

Table 1. Time (in sec) for calculating the OTFs with different methods for primary aberration values given.

\begin{tabular}{|c|c|c|c|c|c|c|}
\hline \multirow[t]{2}{*}{ Aberration } & \multirow[t]{2}{*}{ Value } & \multicolumn{3}{|c|}{ DOTF } & \multicolumn{2}{|c|}{ GOTF } \\
\hline & & NI & DA & FT & NI & FT \\
\hline Defocus: $B_{d} \rho^{2}$ & $B_{d}=1.4$ & 0.26 & 0.04 & 0.033 & 0.47 & 0.031 \\
\hline \multicolumn{7}{|l|}{ Spherical } \\
\hline Minimum $\sigma_{w}: A_{s}\left(\rho^{4}-\rho^{2}\right)$ & $A_{s}=2.3$ & 0.27 & 0.046 & 0.030 & 0.55 & 0.074 \\
\hline Minimum $\sigma_{s}: A_{s}\left[\rho^{4}-(4 / 3) \rho^{2}\right]$ & $A_{S}=1.2$ & 0.17 & 0.053 & 0.029 & 0.53 & 0.079 \\
\hline \multicolumn{7}{|l|}{ Astigmatism: $\quad A_{a} \rho^{2}\left(\cos ^{2} \theta-1 / 2\right)$} \\
\hline$\phi=0, \pi / 2$ & $A_{a}=2.8$ & 0.16 & 0.060 & 0.026 & 0.53 & 0.028 \\
\hline$\phi=\pi / 4$ & $A_{a}=1$ & 0.17 & 0.072 & 0.046 & 0.57 & 0.047 \\
\hline \multicolumn{7}{|l|}{ Coma: $A_{c} \rho^{3} \cos \theta$} \\
\hline$\phi=0$ & $A_{c}=1$ & 0.14 & 0.04 & 0.033 & 0.50 & 0.080 \\
\hline$\phi=\pi / 2$ & $A_{c}=2$ & 0.23 & 0.037 & 0.030 & 0.48 & 0.048 \\
\hline$\phi=\pi / 4$ & $A_{c}=0.7$ & 0.19 & 0.041 & 0.026 & 0.57 & 0.066 \\
\hline
\end{tabular}

\subsection{Imaging System}

Table 2 shows the times for calculating the DOTF and GOTF for the $\mathrm{f} / 2.5$ double Gauss lens, both on axis and at $12^{\circ}$ off axis. Again, the calculation of the GOTF is not any faster than that of the DOTF. Overall, the fastest way to calculate the DOTF is by FT of the PSF, and by FT of the spot diagram for the GOTF.

\section{DISCUSSION AND CONCLUSIONS}

The OTF is a common tool these days for analyzing and specifying the optical quality of a system. The DOTF takes into account the diffraction effects of the system aperture on wave propagation, and provides useful information on how the system images extended objects. However, as discussed previously [1,2], when the amount of aberration in the system is relatively large, the GOTF approximates the DOTF reasonably well. There has been a general notion that calculating theGOTF may be quicker than calculating the DOTF. In this paper, we have shown that this is not true. 
Table 2. Time (in sec) for calculating the OTFs by various methods for the on-axis and $12^{\circ}$ off-axis imaging by the $\mathrm{f} / 2.5$ double Gauss lens.

\begin{tabular}{lccccc}
\hline \multirow{2}{*}{ Input Parametrs } & \multicolumn{3}{c}{ DOTF } & \multicolumn{3}{c}{ GOTF } \\
\hline & NI & DA & FT & NI & FT \\
\cline { 2 - 6 } on-axis $\left(\sigma_{w}=0.4, \sigma_{s}=4.7\right)$ & 0.34 & 0.06 & 0.055 & 0.5 & 0.078 \\
$\mathbf{1 2}^{\mathbf{0}}$ off-axis $\left(\sigma_{w}=0.7, \sigma_{s}=7.0\right)$ & & & & & \\
$\phi=0$ & 0.33 & 0.061 & 0.054 & 0.56 & 0.12 \\
$\phi=\pi / 2$ & 0.27 & 0.061 & 0.054 & 0.53 & 0.093 \\
\hline
\end{tabular}

Using various methods for calculating the two OTFs, Tables 1 and 2 show that calculating them by use of Eqs. (1) and (3) is too slow to be practical, especially, for repetitive calculations, as in design tolerancing at various wavelengths and field angles. However, we have demonstrated with numerical examples that the DOTF calculated by FT of the PSF is faster than calculating the GOTF by FT of the spot diagram. Thus, calculating the GOTF does not offer any advantage. The DOTF calculated by FT of the PSF is available in all major current optical design software on the market. They take advantage of the well-known and efficiently designed Fast Fourier Transform (FFT) algorithm. Based on the study presented in this paper, our general conclusion is that the GOTF calculation, which at best is only an approximation of the DOTF, does not offer any advantage of speed. Hence, a lens designer might as well calculate the true representation of the OTF, namely, the DOTF.

In a separate paper, we discuss the merits of calculating GOTF, and conclude that it does not offer any benefit of speed or accuracy over the DOTF [13].

\section{REFERENCES}

[1] V. N. Mahajan and J. A. Díaz "Comparison of geometrical and diffraction optical transfer functions," Proc. SPIE 9578, 957806 (2015).

[2] V. N. Mahajan and J. A. Díaz "Comparison of geometrical and diffraction imaging in the space and frequency domains," Appl. Opt. 55, 3241-3250 (2016).

[3] CODE V is a registered trademark from Synopsys.

[4] ZEMAX is a registered trademark from Radiant Zemax LLC.

[5] OSLO is a registered trademark from Lambda Research Inc.

[6] SYNOPSIS is a registered trademark from Optical Systems Design, Inc.

[7] V. N. Mahajan, Optical Imaging and Aberrations, Part II: Wave Diffraction Optics, 2nd ed., SPIE Press, Bellingham, WA (2012).

[8] K. Miyamoto, "Image evaluation by spot diagram using a computer," Appl. Opt. 2, 1247-1250 (1963).

[9] V. N. Mahajan, Optical Imaging and Aberrations, Part I: Ray Geometrical Optics, SPIE Press, Bellingham, WA (1998).

[10] Wolfram Research, Inc, Mathematica, Version 11.0, Champaign, Illinois (2016).

[11] M. Laikin, Lens Design, 4th ed., CRC Press, Boca Raton, (2007g), pp. 79-81.

[12] Wolfram Research, Inc, Advanced Numerical Integration In Mathematica, Wolfram Media. Champaign, Illinois (2008).

[13] J. A. Díaz and V. N. Mahajan, "Geometrical optical transfer function: Is it worth calculating it?," accepted for publication in Appl. Opt. (2017). 